\title{
Cardiac tamponade complicating leukaemia: immediate chemotherapy or pericardiocentesis?
}

\author{
Wing-Hung Leung, Yau-Ting Tai, Chu-Pak Lau, Cheuk-Kit Wong, Chun-Ho \\ Cheng and Tai-Kwong Chan
}

Department of Medicine, University of Hong Kong, Queen Mary Hospital, Pokfulam Road, Hong Kong.

\begin{abstract}
Summary: Although leukaemic infiltration of the pericardium is frequently observed at post-mortem, clinically evident cardiac tamponade is rare. Two cases of cardiac tamponade complicating leukaemia are presented. One patient had cardiac tamponade as the initial presentation of acute lymphoblastic leukaemia and experienced complete resolution of the pericardial effusion within 6 days after chemotherapy without therapeutic pericardiocentesis. The other patient with chronic myeloid leukaemia developed cardiac tamponade requiring pericardiocentesis as the first sign of acute blastic transformation. The roles of early chemotherapy and pericardiocentesis in managing this complication are discussed.
\end{abstract}

\section{Introduction}

Leukaemia involves all organs and tissues of the body. Leukaemic infiltration of the pericardium has been documented frequently at post-mortem examinations. ${ }^{1,2}$ Clinically, however, pericardial effusion with cardiac tamponade is rare and only isolated case reports have been described..$^{3-5}$ In all the reported cases, therapeutic pericardiocentesis was required for relief of cardiac tamponade with the risk of bleeding since these patients often had deranged haemostasis.

This report details two patients with leukaemia presenting with cardiac tamponade. To our knowledge, the rapid resolution of cardiac tamponade induced by chemotherapy alone in our first patient and the development of cardiac tamponade as the first sign leukaemia (CML) in the second patient have not been previously described. The role of early chemotherapy and pericardiocentesis will be discussed.

\section{Case reports}

\section{Case 1}

A 45 year old previously healthy Chinese female was admitted for fever and cough for 2 weeks as well as retrosternal chest pain which worsened on deep inspiration for 3 days. She also noticed breathlessness on exertion on the day of admission. On examination, the patient was pale, not in respiratory distress and running a fever of $37.8^{\circ} \mathrm{C}$. The heart rate was 120 beats/min with evidence of pulsus paradoxus and

Correspondence: Wing-Hung Leung, M.B., M.R.C.P. Accepted: 6 April 1989 the blood pressure was $100 / 60 \mathrm{mmHg}$. The jugular venous pressure was elevated up to the angle of the jaw. The cardiac apex was ill-defined and heart sounds were faint. There was no murmur or pericardial friction rub. Abdominal examination revealed $4 \mathrm{~cm}$ hepatomegaly and a spleen tip. Chest radiograph revealed globular cardiac silhouette and a right pleural effusion. Electrocardiogram (ECG) showed generalized diminished voltage and sinus tachycardia. An echocardiogram revealed a large pericardial effusion with gross cardiac oscillation and diastolic collapse of right ventricle and atrium. Laboratory investigations showed haemoglobin $7.3 \mathrm{~g} / \mathrm{dl}$, white blood cell (WBC) count $7.3 \times 10^{9} / 1$ with $79 \%$ blasts, platelet count $45 \times 10^{9} / 1$. The prothrombin time and activated partial thromboplastin time were normal. Bone marrow aspirate and cytochemical and immunocytochemical studies confirmed the diagnosis of T-cell acute lymphocytic leukaemia (ALL). Smears on the pleural fluid aspirated with platelet infusion cover using the Gram and Ziehl-Neelsen stains, and cultures for aerobic, anaerobic, and tubercle bacilli were negative. It showed a WBC count of $12.8 \times 10^{9} / 1$ with $90 \%$ lymphoblasts.

She was transfused and was promptly started on systemic chemotherapy comprising vincristine, cyclophosphamine, prednisone and daunorubicin. Therapeutic pericardiocentesis was withheld owing to the relative lack of symptoms, while the patient was closely monitored with serial chest radiographs and echocardiography. There was symptomatic improvement within 48 hours with disappearance of pulsus paradoxus and normalization of jugular venous pressure. The blood pressure went up to $120 / 70 \mathrm{mmHg}$, 
the pulse rate decreased to $90 / \mathrm{min}$. Echocardiogram 2 days after chemotherapy revealed reduction in size of pericardial effusion and absence of diastolic compression of right ventricle. The temperature returned to normal on day 4 and another echocardiogram on day 6 revealed complete resolution of the pericardial effusion. Chest radiograph revealed decreased cardiothoracic ratio and resolution of the pleural effusion. The patient remained in haematological remission and there was no evidence of recurrence of pericardial effusion 6 weeks afterwards.

\section{Case 2}

A 19 year old female with Philadelphia chromosome positive CML was maintained in the chronic phase for the past 5 years with busulphan. She was admitted into hospital because of rapid onset of sharp retrosternal chest pain, epigastric pain and shortness of breath over 24 hours. Physical examination showed she had central cyanosis and cold peripheries. The blood pressure was $80 / 50 \mathrm{mmHg}$ and pulse rate was 140 / min, with marked pulsus paradoxus. The jugular venous pressure was raised and Kussmaul's sign was elicited. The cardiac impulse was ill-defined and the heart sounds were distant. A typical pericardial friction rub was heard. The liver was palpable $4 \mathrm{~cm}$ below the right costal margin and was tender. The spleen was palpable $10 \mathrm{~cm}$ below the left costal margin. Chest radiograph revealed cardiomegaly. Electrocardiogram revealed sinus tachycardia and diffuse nonspecific $T$ wave inversion. Echocardiogram confirmed the presence of pericardial effusion with diastolic collapse of right ventricle and right atrium. Haemoglobin was $10.8 \mathrm{~g} / \mathrm{dl}$, platelet count $126 \times 10^{9} / 1$ and WBC count $39.4 \times 10^{9} / 1$ with blasts $15 \%$. Cytochemical and immunophenotyping studies failed to define the lineage of the blast cells.

In view of the severity of her symptoms, pericardiocentesis was performed via the subxiphoid approach. Two hundred millilitres of straw-coloured fluid was obtained and the patient's clinical status improved dramatically. Analysis of the pericardial fluid showed protein $55 \mathrm{~g} / \mathrm{l}$, glucose $7.8 \mathrm{mmol} / \mathrm{l}$, WBC count $5.6 \times 10^{9} / 1$ with $40 \%$ blast cells. Cultures for bacteria, fungus and acid-fast bacilli were repeatedly negative. Bone marrow trephine biopsy confirmed CML in blastic transformation. She was given hydroxyurea with initial response. The WBC count decreased to $13.7 \times 10^{9} / 1$. Echocardiogram showed a residual rim of pericardial effusion.

Four weeks later, however, she was readmitted again because of epigastric pain, shortness of breath as well as generalized erythematous plaque-like lesions over the face and trunk. Another chest radiograph and echocardiogram showed recurrence of massive pericardial effusion. The WBC count rose to
$41.4 \times 10^{9} / 1$ with $39 \%$ blasts. In view of the recurrent nature of her pericardial effusion, pericardotomy with drainage was performed; $375 \mathrm{ml}$ of blood-stained fluid $c$ was released. Pericardial biopsy revealed a thickened fibrotic pericardium with perivascular infiltration of inflammatory cells and fibroblasts. There was noo definite evidence of leukaemic infiltration nor infec-음 tion. Biopsy of the cutaneous erythematous plaque $\overline{\frac{\bar{\omega}}{\sigma}}$ showed marked leukaemic infiltration of the dermis. $\stackrel{\circ}{\circ}$ She was given chemotherapy comprising vincristine, cytosine arabinoside and thioguanine. There was no reaccumulation of pericardial effusion and the pericar- $-\circ$ dial drain was removed after 4 days. However, she died $\overrightarrow{\vec{\omega}}$ 4 weeks later because of fulminant pneumonia. Post- $\omega$ mortem examination was refused.

\section{Discussion}

In a large autopsy study of 420 patients with acute $y$ leukaemia, Roberts et al. observed leukaemic $\omega$ infiltration in the hearts of 156 patients $(37 \%), 99$ of whom showed pericardial infiltrates. ${ }^{1}$ However, it is unusual for the cardiac involvement to be manifested clinically and even rarer to be the initial presentation ${ }_{0}^{\circ}$ of leukaemia.

Chu et al in 1983 reviewed the literature and fou 17 reported patients with leukaemia who had manifes tations of pericarditis with cardiac tamponade. ${ }^{3}$ They. ${ }^{\circ}$ included 9 patients with ALL, 5 with acute myeloblas-0 tic leukaemia, 2 with CML and one with chronic lymphocytic leukaemia. Cassis et al. described ā̄ 45 year old man with massive haemopericardium as the initial manifestation of CML. ${ }^{4}$ One similar patient $\stackrel{\mathbb{Q}}{\mathscr{Q}}$ with ALL was described by Mancuso et al. ${ }^{5}$ Review of $\overrightarrow{\vec{P}}$ all these cases showed that all patients required $\frac{}{3}$ pericardiocentesis in addition to chemotherapy and/or $\vec{F}$ radiotherapy for control of the pericardial effusion.

The first patient in this report showed several interesting features. First, the cardiac tamponade was 3 . the initial presentation of her ALL. Second, the blast $\overline{5}$ cells in this patient were of T-cell origin. T-cell ALL with cardiac tamponade has also been described by 0 Mancuso et al..$^{5}$ The cell types in other reported cases $₹$ of ALL with cardiac tamponade were not specified. 옥 T-cell ALL is well known to have mediastinal involve- $\rightarrow$ ment, but whether T-cell ALL has a predilection for pericardial infiltration remains to be seen. Third, the pericardial effusion responded dramatically to $\mathrm{N}$ chemotherapy, with complete resolution within $\tilde{O}$ 6 days. Such rapid resolution of cardiac tamponade in $N$ leukaemia induced by chemotherapy alone has not 0 been previously reported.

Cardiac tamponade complicating CML is especially $\stackrel{0}{\overparen{D}}$ rare $, 3,4$ and occurred in the chronic phase of the disease $\stackrel{f}{?}$ in all the reported cases. Its occurrence as the first 7 clinical manifestation of acute blastic transformation, 
as in our second patient, has not been described previously.

All the evidence points towards malignant infiltration as the cause of the pericardial effusion in our patients. In the first patient, the pericardial effusion resolved rapidly with chemotherapy, and the coexistent pleural effusion was proven to be leukaemic in origin. In the second patient, the pericardial fluid showed blast cells, and was repeatedly culture negative. The accompanying extensive leukaemic infiltration of the skin further supported this. Moreover, the recurrence of the effusion correlated with the activity of the blastic stage of the disease. A negative pericardial biopsy does not disprove the diagnosis, as leukaemic pericardial infiltration may be scattered. ${ }^{1}$

\section{References}

1. Roberts, W.C., Bodey, G.P. \& Wertlake, P.T. The heart in acute leukemia: a study of 420 autopsy cases. Am J Cardiol 1968, 21: $388-412$.

2. Bierman, H.R., Perkins, E.K. \& Orega, P. Pericarditis in patients with leukemia. Am Heart J 1952, 43: 413-422.

3. Chu, J.Y., Demello, D. O'Connor, D.M., Chen, S.C., Gale, G.B. Pericarditis as presenting manifestation of acute nonlymphocytic leukemia in a young child. Cancer 1983, 52: 322-324.

4. Cassis, N. Jr, Porterfield, J., Rogers, C.S. II, Shah, S. \& Storey, E. Massive hemopericardium as the initial manifestation of chronic myelogenous leukemia. Arch Intern Med 1982, 142: 2193-2194.
In conclusion, pericardial effusion should be considered in patients with leukaemia who experience sudden onset of cardiac symptoms. Its management should however be individualized. In those in whom the pericardial effusion is the initial presentation of the disease and provided the clinical status of the patients allows, pericardiocentesis may be withheld and systemic chemotherapy promptly administered, thus avoiding the potential complications of pericardiocentesis in patients with bleeding tendency. Pericardiocentesis or pericardotomy is required when the pericardial effusion is resistant to chemotherapy, when patient symptoms are severe, and when infection has to be excluded. Radiotherapy and intrapericardial chemotherapy may have to be considered in resistant cases of leukaemic pericardial effusion. ${ }^{6.7}$

5. Mancuso, L., Marchi, S., Giuliano, P. \& Pitrolo, F. Cardiac tamponade as first manifestation of acute lymphoblastic leukemia in a patient with echographic evidence of mediastinal lymph node enlargement. Am Heart $J$ 1985, 110: 1303-1304.

6. Terry, L.N. Jr \& Klingerman, M.N. Pericardial and myocardial involvement by lymphoma and leukemias: the role of radiotherapy. Cancer 1969, 25: 1003-1008.

7. Press, O.W. \& Livingston, R. Management of malignant pericardial effusion and tamponade. JAMA 1987, 256: 2301-2307. 